\title{
ESTUDO DO CRESCIMENTO DE ESPÉCIES DE ÁRVORES SEMIDECÍDUAS EM UMA ÁREA CILIAR REVEGETADA ${ }^{1}$
}

\author{
Melina Teles França Sampaio², Marcelo Polo e Wilson Barbosa ${ }^{4}$
}

\begin{abstract}
RESUMO - Este trabalho foi realizado com os objetivos de avaliar o crescimento das espécies e identificar o seu padrão de crescimento e discutir sobre a eficiência do método em relação ao processo de restauração vegetal. O levantamento dos dados foi realizado 32 meses após o plantio das mudas. Para a amostragem foi empregado o método de amostragem de área fixa com parcelas, sendo utilizadas cinco áreas amostrais, que foram selecionadas de modo a abranger toda a extensão reflorestada, contendo 200 indivíduos/área amostral. Foram realizadas, em todas as plantas amostradas do estrato arbóreo com altura superior a $1 \mathrm{~m}$, medições de altura e de diâmetro. Os dados foram analisados como delineamento em blocos casualizados (DBC) e submetidos à análise de variância, seguida do teste de Scott \& Knott a 5\% de significância, utilizando-se o programa SISVAR. Por fim, concluiu-se que espécies pioneiras ou que apresentam ciclo de vida curto são consideradas ideais para a implantação na fase inicial do reflorestamento, devido ao seu rápido crescimento. Entretanto, devem ter seu manejo associado a espécies não pioneiras ou perenes.
\end{abstract}

Palavras-chave: Restauração vegetal, Recuperação ambiental e Espécies pioneiras.

\section{ESTUDY OF THE GROWTH OF TREE SEMIDECIDUOUS SPECIES IN AN RIPARIAN AREA REVEGETATED}

\begin{abstract}
The objective of this study was to evaluate the species growing, to identify their growth pattern and to discuss the method quality in relation to plant reforestation. The survey was conducted after 32 months of planting seedlings, through sampling of fixed area by using plots, in which five sampling areas, selected to cover the entire length reforested, containing 200 individuals/sample area was used. Height and diameter were measured in all sampled plants, with a height greater than 1 (one) meter. Data were analyzed as a randomized block lineation (RBL), subjected to analysis of variance followed by Scott \& Knott test at $5 \%$ of significance level, using the program SISVAR. Finally, it was concluded that pioneer species or those that have a short life cycle are considered ideal for deployment in early of reforestation due to fast growth; however they should have their management associated with not pioneer species or perennials species.
\end{abstract}

Keywords: Environmental recovery, Plant restoration and Pioneer species.

\section{INTRODUÇÃO}

Estudos têm evidenciado que a intervenção humana visando à revegetação de áreas degradadas é de grande importância para que estas consigam recuperar a resiliência, já que o processo natural de sucessão florestal pode simplesmente não ocorrer ou ser extremamente lento (CARPANEZZI et al., 2004).
Segundo Pickett et al. (1987) e Rodrigues e Gandolfi (1998), para que a restauração aconteça naturalmente são necessárias condições favoráveis à revegetação da área, como a presença de sementes no solo (banco de sementes) ou a chegada de sementes por meio da dispersão. Portanto, a restauração de ecossistemas está além da simples cobertura vegetal do solo (RODRIGUES; GANDOLFI, 2001), e as atividades nos

\footnotetext{
${ }^{1}$ Recebido em 09.12.2011 aceito para publicação em 04.06.2012..

${ }^{2}$ Programa de Pós-Graduação em Ecologia e Tecnologia Ambiental, UNIFAL/MG, Brasil. E-mail: <melinafsampaio@ hotmail.com>.

${ }^{3}$ Instituto de Ciências da Natureza, Laboratório de Biotecnologia Ambiental \& Genotoxicidade, UNIFAL/MG, Brasil. E-mail: <marcelo.polo@unifal-mg.edu.br>.

${ }^{4}$ Instituto Agronômico de Campinas, Centro Experimental Central, IAC/SP, Brasil. E-mail: <wbarbosa@iac.sp.gov.br>.
} 
projetos de restauração de áreas alteradas devem ocorrer com o intuito principal de acelerar o processo de regeneração natural (BERTONI; DICKFELDT, 2007).

Até o início dos anos 80 , a restauração era fundamentada no plantio de árvores sem qualquer critério ecológico para a escolha e combinação das espécies, pois havia pouco conhecimento em relação aos processos ecológicos na dinâmica das Florestas Estacionais Semidecíduas (RODRIGUES; GANDOLFI, 1996).

Com o passar do tempo, observou-se que mudanças na metodologia eram necessárias para que as florestas restauradas pudessem edificar-se. Muitos equívocos foram sendo corrigidos até que fosse adotada a implantação direta de linhas de pioneiras e não pioneiras, mas essa técnica também passou a ser questionada em relação à efetiva ocupação da área, já que espécies como as embaúbas (Cecropia spp.) e os guapuruvus (Schizolobium spp.), apesar de crescerem rapidamente, não promovem a cobertura do solo esperada na linha das pioneiras (NAVE, 2005; NAVE; RODRIGUES, 2007). Dessa forma, foi adotado um novo modelo de restauração florestal, no qual as espécies nativas são distribuídas em grupos de Preenchimento e em grupos de Diversidade. O primeiro grupo é composto por espécies que se destacam no crescimento e cobertura de copa que irá proporcionar o rápido fechamento da área plantada. Já o grupo de Diversidade é constituído por espécies com características que diferem das espécies do primeiro grupo, mas que irão garantir a perpetuação da área plantada de forma sustentável (RODRIGUES et al., 2009).

Diante ao exposto, conhecer as características das espécies utilizadas no processo de restauração vegetal de uma área é essencial para não comprometer o sucesso do projeto (RODRIGUES; GANDOLFI, 2000; ENGEL; PARROTTA, 2003), e o monitoramento dessas áreas é importante, pois só assim será possível avaliar o processo de restauração, definir medidas de manejo, verificar a eficiência do método e aperfeiçoar os modelos de restauração (IGNÁCIO et al., 2007).

Com a finalidade de ampliar o conhecimento sobre o assunto, o objetivo deste trabalho foi conhecer o padrão de crescimento das diferentes espécies utilizadas no reflorestamento da área, em que se podem verificar o desempenho delas e a eficiência do método em relação ao processo de restauração vegetal, levando em conta os parâmetros de avaliação adotados.

\section{MATERIAL E MÉTODOS}

\subsection{Caracterização da área}

Este estudo foi conduzido no Centro Experimental Central (CEC) do Instituto Agronômico de Campinas (IAC), situado na cidade de Campinas, entre as latitudes $22^{\circ} 46^{\prime} 10^{\prime \prime} \mathrm{S}$ a $23^{\circ} 03^{\prime} 11^{\prime \prime} \mathrm{S}$ e as longitudes $46^{\circ} 50^{\prime} 19^{\prime \prime} \mathrm{O}$ a $47^{\circ} 13^{\prime} 05^{\prime \prime} \mathrm{O}$, a uma altitude de $674 \mathrm{~m}$. O clima da área apresenta temperatura média anual de $21^{\circ} \mathrm{C}$, com regime de chuvas típico de zonas tropicais de baixa altitude, com verão chuvoso e inverno seco e vegetação natural de Floresta Estacional Semidecídua. Os dados referentes às condições climáticas, obtidos pelo Centro de Ecofisiologia e Biofísica do Instituto Agronômico (IAC), permitiram identificar o clima como do tipo Cwa, segundo o sistema de Köppen (1948), ou seja, clima mesotérmico de inverno seco e precipitação anual de $1.371 \mathrm{~mm}$ e déficit hídrico anual na ordem de $50 \mathrm{~mm}$. Segundo o mapeamento geomorfológico de Campinas, realizado pelo Instituto de Pesquisas Tecnológicas (IPT), a região do CEC está localizada em uma área de colinas amplas, com vales acumulativos e abertos.

Desde 1920, o IAC realiza diversas experimentações agrícolas na área do CEC, também conhecida como Fazenda Santa Elisa, que conta hoje com uma área de aproximadamente 692 ha (GRAZIANO, 2006). Com a finalidade de compensação por passivos ambientais, empresas privadas desenvolveram, nos meses de dezembro (2008) e janeiro (2009), a recomposição da vegetação na área ciliar do córrego Santa Elisa, situado nas dependências do CEC, plantando cerca de 16 mil mudas de árvores, em sua maioria de espécies nativas.

\subsection{Método de amostragem}

Para o levantamento dos dados foi adotado o método de amostragem de área fixa com emprego de parcelas (MANTOVANI et al., 2005), sendo utilizadas cinco áreas amostrais, que foram selecionadas de modo a abranger toda a extensão reflorestada, contendo 200 indivíduos/ área amostral. Ao todo, foram quatro áreas com 10 linhas contendo 20 indivíduos em cada linha e uma área com oito linhas contendo 25 indivíduos em cada linha. As medições de altura e diâmetro (à altura do solo) foram realizadas em todos os indivíduos do estrato arbóreo, com altura superior a $1 \mathrm{~m}$, pertencentes às cinco áreas amostradas. O levantamento dos dados foi realizado nos meses de setembro e outubro de 2011, aos 32 meses após o plantio. 


\subsection{Parâmetros de avaliação}

Em relação à análise estatística, os dados amostrados, que tratam de delineamento em blocos casualizados (DBC), foram submetidos à análise de variância, seguida do teste de Scott \& Knott a 5\% de significância, utilizando-se o programa SISVAR. O objetivo foi verificar se havia diferença estatística significativa entre as espécies em relação ao crescimento (altura e diâmetro).

\section{RESULTADOS}

Foram amostrados 1.000 indivíduos do estrato arbóreo em uma área com menos de 1 ha (0,352 h). Em relação à riqueza de espécies, foi possível amostrar
31 espécies, pertencentes a 15 famílias, entre elas a grande família das Leguminosae, que apresentou três subfamílias (Tabela 1).

As análises de variância, realizadas comparando altura e diâmetro de todas as espécies, foram significativas em todos os testes $(\mathrm{p}<0,0001)$. Em relação à altura, os testes indicaram que Acacia auriculiformis (6,0 m) e Cecropia pachystachya $(5,9 \mathrm{~m})$ apresentaram as maiores médias em altura, sendo significativamente diferentes das demais espécies. Em seguida vieram espécies como Pterogyne nitens (4,8 m), Aloysia virgata (4,6 m), Schinus terebinthifolia $(4,3 \mathrm{~m})$ e Inga cylindrica $(4,3 \mathrm{~m})$, apresentando valores que podem ser considerados como intermediários, já que outras espécies como Cordia

Tabela 1 - Família, nome científico, nome popular e grupo ecológico das espécies amostrada no levantamento realizado em cinco áreas amostrais ao longo da área ciliar do Córrego Santa Elisa, em Campinas, SP.

Table 1 - Family, scientific name, popular name and ecological group of species sampled in the survey conducted in five sampling area in riparian area along Santa Elisa brook in Campinas, SP.

\begin{tabular}{|c|c|c|c|}
\hline Família & Nome científico & Nome popular & $\mathrm{GP}^{*}$ \\
\hline Anacardiaceae & Schinus terebinthifolia & Aroeira-mansa & $\mathrm{P}$ \\
\hline Bignoniaceae & Tabebuia chrysotricha & Ipê-amarelo & NP \\
\hline \multirow[t]{3}{*}{ Bombacaceae } & Bombacopsis glabra & Castanha-do-maranhão & $\mathrm{P}$ \\
\hline & Chorisia speciosa & Paineira & $\mathrm{P}$ \\
\hline & Pseudobombax grandiflorum & Embiriçu & $\mathrm{P}$ \\
\hline Boraginaceae & Cordia superba & Babosa-branca & NP \\
\hline Cecropiaceae & Cecropia pachystachya & Embaúba & $\mathrm{P}$ \\
\hline \multirow[t]{2}{*}{ Euphorbiaceae } & Joannesia princeps & Andá-açu & NP \\
\hline & Croton urucurana & Sagra-d'água & $\mathrm{P}$ \\
\hline \multirow[t]{4}{*}{ Leguminosae-Caesalpinoidae } & Pterogyne nitens & Amendoim & $\mathrm{P}$ \\
\hline & Senna bicapsularis & Canudo-de-pito & $\mathrm{P}$ \\
\hline & Hymenaea stilbocarpa & Jatobá & NP \\
\hline & Peltophorum dubium & Canafístula & $\mathrm{P}$ \\
\hline \multirow[t]{5}{*}{ Leguminosae-Mimosoideae } & Acacia auriculiformis & Acácia & $\mathrm{P}$ \\
\hline & Anadenanthera colubrina & Angico-branco & $\mathrm{P}$ \\
\hline & Enterolobium contortisiliquum & Tamboril & $\mathrm{P}$ \\
\hline & Inga cylindrica & Ingá & $\mathrm{P}$ \\
\hline & Piptadenia gonoacantha & Pau-jacaré & $\mathrm{P}$ \\
\hline \multirow[t]{3}{*}{ Leguminosae-Papilionoideae } & Myroxylon peruiferum & Cabreúva & NP \\
\hline & Citharexylum myrianthum & Pau-de-viola & $\mathrm{P}$ \\
\hline & Erythrina speciosa & Eritrina & $\mathrm{P}$ \\
\hline Lythraceae & Lafoensia pacari & Dedaleiro & $\mathrm{P}$ \\
\hline Myrsinaceae & Rapanea ferrugínea & Capororoca & $\mathrm{P}$ \\
\hline \multirow[t]{2}{*}{ Myrtaceae } & Eugenia uniflora & Pitanga & NP \\
\hline & Psidium guajava & Goiabeira & $\mathrm{P}$ \\
\hline Phytolaccaceae & Gallesia integrifólia & Pau-d'alho & NP \\
\hline Polygonaceae & Triplaris americana & Pau-formiga & $\mathrm{P}$ \\
\hline Rubiaceae & Genipa americana & Jenipapo & NP \\
\hline Tiliaceae & Heliocarpus americanos & Algodoeiro & $\mathrm{P}$ \\
\hline Verbenaceae & Aloysia virgata & Lixa & $\mathrm{P}$ \\
\hline Anacardiaceae & Vitex montevidensis & Tarumã & NP \\
\hline
\end{tabular}

* Grupo ecológico: P = Pioneiras; NP = Não pioneiras (segundo modelo de SWAINE; WHITMORE, 1988). 
superba (1,8 m), Myroxylon peruiferum (1,9 m), Eugenia uniflora (2,0 m) e Erythrina speciosa (2,1 m) apresentaram médias significativamente menores, conforme observado na Tabela 2. Já em relação ao diâmetro, os testes evidenciaram que espécies como Aloysia virgata $(14,9 \mathrm{~cm})$, Cecropia pachystachya $(12,6 \mathrm{~cm})$, Chorisia speciosa $(11,0 \mathrm{~cm})$ e Joannesia princeps $(10,8 \mathrm{~cm})$ fazem parte do grupo das espécies que apresentaram as maiores médias, diferindo significativamente do grupo daquelas com médias menores, composto por espécies como Enterolobium contortisiliquum (2,8 cm), Myroxylon peruiferum (3,1 cm), Eugenia uniflora $(3,6 \mathrm{~cm})$ e Senna bicapsularis $(3,8)$ (Tabela 3$)$.

Tabela 2 - Média de altura (m) das espécies amostradas no levantamento ao longo do córrego Santa Elisa.

Table 2-Mean of the height ( $m$ ) of species sampled in the survey conducted along Santa Elisa brook.

\begin{tabular}{llc}
\hline Espécies & Média & Resultado \\
\hline Acacia auriculiformis & 6.000 & $\mathrm{~A}$ \\
Cecropia pachystachya & 5.920 & $\mathrm{~A}$ \\
Pterogyne nitens & 4.780 & $\mathrm{~B}$ \\
Aloysia virgata & 4.600 & $\mathrm{~B}$ \\
Schinus terebinthifolia & 4.340 & $\mathrm{~B}$ \\
Inga cylindrica & 4.280 & $\mathrm{~B}$ \\
Anadenanthera colubrina & 4.260 & $\mathrm{~B}$ \\
Croton urucurana & 4.140 & $\mathrm{~B}$ \\
Genipa americana & 4.140 & $\mathrm{~B}$ \\
Piptadenia gonoacantha & 4.000 & $\mathrm{~B}$ \\
Joannesia princeps & 3.880 & $\mathrm{~B}$ \\
Chorisia speciosa & 3.780 & $\mathrm{~B}$ \\
Hymenaea stilbocarpa & 3.780 & $\mathrm{~B}$ \\
Heliocarpus americanus & 3.420 & $\mathrm{C}$ \\
Lafoensia pacari & 3.180 & $\mathrm{C}$ \\
Bombacopsis glabra & 3.040 & $\mathrm{C}$ \\
Vitex montevidensis & 3.020 & $\mathrm{C}$ \\
Triplaris brasiliensis & 2.900 & $\mathrm{C}$ \\
Tabebuia chrysotricha & 2.860 & $\mathrm{C}$ \\
Peltophorum dubium & 2.820 & $\mathrm{C}$ \\
Pseudobombax grandiflorum & 2.820 & $\mathrm{C}$ \\
Rapanea ferruginea & 2.760 & $\mathrm{C}$ \\
Citharexylum myrianthum & 2.700 & $\mathrm{C}$ \\
Senna bicapsularis & 2.460 & $\mathrm{C}$ \\
Gallesia integrifolia & 2.440 & $\mathrm{C}$ \\
Enterolobium contortisiliquum & 2.400 & $\mathrm{C}$ \\
Psidium guajava & 2.200 & $\mathrm{C}$ \\
Erythrina speciosa & 2.090 & $\mathrm{C}$ \\
Eugenia uniflora & 1.980 & $\mathrm{C}$ \\
Myroxylon peruiferum & 1.920 & $\mathrm{C}$ \\
Cordia superba & 1.760 & $\mathrm{C}$ \\
\hline Terion & & \\
\hline
\end{tabular}

Médias seguidas de mesma letra não diferem entre si, a $5 \%$ de significância, de acordo com o teste de Scott-Knott.
As análises de variância realizadas comparando altura e diâmetro de espécies consideradas pioneiras e não pioneiras, segundo a classificação de Swaine e Whitmore (1988), foram significativas apenas pelo teste em relação à altura $(\mathrm{p}<0,0072)$. Assim, as espécies pioneiras apresentaram média de altura $(3,6 \mathrm{~m})$ significativamente maior que a média de altura $(2,9 \mathrm{~m})$ das espécies não pioneiras (Tabela 4).

\section{DISCUSSÃO}

Espécies que se destacaram em relação ao crescimento em altura, como Acacia auriculiformis, que também apresentou destaque no levantamento

Tabela 3 - Média de diâmetro $(\mathrm{cm})$ das espécies amostradas no levantamento ao longo do córrego Santa Elisa.

Table 3-Mean of diameter (cm) of species sampled in the survey conducted along Santa Elisa brook.

\begin{tabular}{lcc}
\hline Espécies & Média & Resultado \\
\hline Aloysia virgata & 14.920 & $\mathrm{~A}$ \\
Cecropia pachystachya & 12.560 & $\mathrm{~A}$ \\
Chorisia speciosa & 10.980 & $\mathrm{~A}$ \\
Joannesia princeps & 10.760 & $\mathrm{~A}$ \\
Inga cylindrica & 10.000 & $\mathrm{~A}$ \\
Acacia auriculiformis & 9.980 & $\mathrm{~A}$ \\
Heliocarpus americanus & 9.860 & $\mathrm{~A}$ \\
Anadenanthera colubrina & 9.260 & $\mathrm{~A}$ \\
Croton urucurana & 8.340 & $\mathrm{~A}$ \\
Genipa americana & 8.300 & $\mathrm{~A}$ \\
Bombacopsis glabra & 8.080 & $\mathrm{~A}$ \\
Erythrina speciosa & 7.900 & $\mathrm{~A}$ \\
Schinus terebinthifolia & 7.550 & $\mathrm{~A}$ \\
Cordia superba & 6.620 & $\mathrm{~B}$ \\
Hymenaea stilbocarpa & 6.560 & $\mathrm{~B}$ \\
Citharexylum myrianthum & 6.440 & $\mathrm{~B}$ \\
Piptadenia gonoacantha & 6.400 & $\mathrm{~B}$ \\
Tabebuia chrysotricha & 6.400 & $\mathrm{~B}$ \\
Pseudobombax grandiflorum & 6.160 & $\mathrm{~B}$ \\
Gallesia integrifolia & 5.700 & $\mathrm{~B}$ \\
Pterogyne nitens & 5.540 & $\mathrm{~B}$ \\
Rapanea ferruginea & 4.580 & $\mathrm{~B}$ \\
Triplaris brasiliensis & 4.500 & $\mathrm{~B}$ \\
Psidium guajava & 4.440 & $\mathrm{~B}$ \\
Peltophorum dubium & 4.340 & $\mathrm{~B}$ \\
Vitex montevidensis & 4.140 & $\mathrm{~B}$ \\
Lafoensia pacari & 4.090 & $\mathrm{~B}$ \\
Senna bicapsularis & 3.800 & $\mathrm{~B}$ \\
Eugenia uniflora & 3.580 & $\mathrm{~B}$ \\
Myroxylon peruiferum & 3.140 & $\mathrm{~B}$ \\
Enterolobium contortisiliquum & 2.840 & $\mathrm{~B}$ \\
\hline Medissguas & & \\
\hline
\end{tabular}

Médias seguidas de mesma letra não diferem entre si, a 5\% de significância, de acordo com o teste de Scott-Knott. 
Tabela 4 - Média de altura (m) de espécies pioneiras e não pioneiras amostradas no levantamento ao longo do córrego Santa Elisa.

Table 4-Mean of height ( $m$ ) of pioneer and not pioneers species sampled in the survey conducted along Santa Elisa brook.

\begin{tabular}{lcc}
\hline Grupo Ecológico & Média & Resultado \\
\hline Pioneiras & 3.586 & $\mathrm{~A}$ \\
Não Pioneiras & 2.864 & $\mathrm{~B}$ \\
\hline
\end{tabular}

Médias seguidas de mesma letra não diferem entre si, a 5\% de significância, de acordo com o teste de Scott-Knott.

realizado por Davide e Faria(1997), podem ser consideradas adequadas para a recuperação de áreas degradadas (FERREIRA etal., 1990; DIAS etal., 1991), por apresentarem crescimento rápido e se manterem por vários anos no ambiente(UGALDE, 1985). Porém, espécies como Cecropia pachystachya, que, apesar de crescimento rápido, apresentam ciclo de vida curto (VICENTINI et al., 2008), podem não estar presentes na floresta futura. Dessa forma, espécies com características semelhantes às da Cecropia pachystachya devem ter seu manejo associado a espécies com ciclo de vida perene, para que a área reflorestada tenha maiores chances de se manter com o passar dos anos.

Ainda analisando os dados apresentados pela espécie Acacia auriculiformis, observou-se que essa espécie se destaca tanto em relação à média de altura quanto em relação à média em diâmetro. Segundo Sturion e Antunes (2000), a relação entre a altura e diâmetro do indivíduo em geral retrata o acúmulo de reservas, e com o passar dos anos o crescimento diamétrico tende a aumentar (LIMA, 2009).

Em relação à grande família das Leguminosae, constatou-se que seis espécies, das 12 dessa família que foram amostradas durante o levantamento, integraram os dois primeiros grupos que se destacaram em relação às médias de altura. Essa informação corrobora Chada et al. (2004), que consideram as espécies da família Leguminosae fundamentais para a recuperação de áreas degradadas, devido à capacidade de suas raízes em se associarem a fungos micorrízicos e bactérias do gênero Rhizobium.

\section{CONCLUSÃO}

Espécies pioneiras, ou que apresentam ciclo de vida curto, são consideradas mais adequadas para implantação na fase inicial do reflorestamento por terem rápido crescimento, entretanto devem ter seu manejo associado a espécies não pioneiras ou perenes, para garantir não só a perpetuação da área reflorestada, mas também a diversidade de espécies florestais.

A área ciliar onde foram coletados os dados apresentados neste artigo deve continuar sendo monitorada, para que o sucesso da restauração vegetal se confirme.

\section{AGRADECIMENTOS}

Ao IAC, pela oportunidade concedida para a realização desta pesquisa; e ao Dr. José Eduardo de Arruda Bertoni, pelo auxílio técnico.

\section{REFERÊNCIAS}

BERTONI, J. E.A.; DICKFELDT, E. P. Plantio de Myracrodruon urundeuva Fr. All. (aroeira) em área alterada de floresta: desenvolvimento das mudas e restauração florestal. Revista Instituto Florestal, v.19, n.1, p.31-38, 2007.

CARPANEZZI, A. A. Espécies pioneiras para recuperação de áreas degradadas: a observação de laboratórios naturais. In: CONGRESSO FLORESTAL BRASILEIRO, 6 ., 2004, Campos do Jordão. Anais... Campos do Jordão: 2004. v.3.

CHADA, S. S.; CAMPELLO, E. F. C.; FARIA, S. M. Sucessão vegetal em uma encosta reflorestada com leguminosas arbóreas em Angra dos Reis, RJ. Revista Árvore, v.28, n.6, p.801-809, 2004.

DAVIDE, A. C.; FARIA, J. M. R. Revegetação de área de empréstimo da Usina Hidrelétrica de Camargos (CEMIG). In: SIMPÓSIO NACIONAL DE RECUPERAÇÃO DE ÁREAS DEGRADADAS, 1997, Viçosa, MG. Anais... Viçosa, MG: SOBRADE/UFV/DPS/DEF, 1997.

DIAS, E. D.; ALVAREZ V., V. H.; BRIENZA JÚNIOR, S. Formação de mudas de Acacia mangium. Resposta a nitrogênio e potássio. Revista Árvore, v.15 p 11-22, 1991.

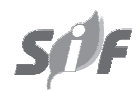

Revista Árvore, Viçosa-MG, v.36, n.5, p.879-885, 2012 
ENGEL, V. L.; PARROTA, J. A. Definindo a restauração ecológica: tendências e perspectivas mundiais. In: KAGEYAMA, P. Y. et al. (Ed.) Restauração ecológica de ecossistemas naturais. Botucatu: Fundação de Estudos e Pesquisas Agrícolas e Florestais, 2003. p.3-25.

FERREIRA, C. A. et al. Acacia mangium: uma nova opção para reflorestamento. In: CONGRESSO FLORESTAL BRASILEIRO, Campos do Jordão, 1990. Anais... Campos do Jordão: SBS, 1990. p.564-568.

GRAZIANO, A. T. Jardim Botânico Santa Elisa: Um jardim botânico agrícola no Brasil. 2006, 160 p. Dissertação (Mestrado em Arquitetura e Urbanismo) - FAU, Universidade de São Paulo, São Paulo, 2006.

IGNÁSIO, E. D.; ATTANÁSIO, C. M.; TONiATO, M. T. Z. Monitoramento de plantios de restauração de florestas ciliares: Microbacia do Ribeirão São João, Mineiros do Tietê, SP. IF Série Regional.,n. 31, p.219223, 2007. Disponível em:<http:// www.iflorestal.sp.gov.br/publicacoes/ serie_registros/IFSerReg31/219-223.pdf>. Acessado em: 2011-10-03.

KÖPPEN, W. Climatologia: con un estudio de los climas de la tierra. México: Fondo de Cultura Econômica, 1948.

LIMA, J. A.; SANTANA, D. G.; NAPPO, M. E. Comportamento inicial de espécies na revegetação da mata de galeria na Fazenda Mandaguari, em Indianópolis, MG. Revista Árvore, 2009. Disponível em http:// www.scielo.br/scielo.php?script $=$ sci_ arttext\&pid=S0100-

$67622009000400011 \& \operatorname{lng}=$ pt\&nrm =iso. Acessado em 16 ago. 2011.

\section{MANTOVANI, A. et al. Inventário e} manejo florestal: amostragem, caracterização de estádios sucessionais na vegetação catarinense e manejo do palmiteiro (Euterpe edulis) em regime de rendimento sustentável. Florianópolis: Núcleo de Pesquisas em Florestas Tropicais, 2005.

Revista Árvore, Viçosa-MG, v.36, n.5, p.879-885, 2012
NAVE, A. G.; RODRIGUES, R. R. Combination of species into filling and diversity groups as forest restoration methodology. In: RODRIGUES, R.R.; MARTINS, S.V.; GANDOLFI, S. (Org.). High diversity forest restoration in degraded areas: methods and projects in Brazil. New York: Nova Science Publishers, 2007. p.197-206.

NAVE, A. G. Banco de sementes autóctone e alóctone, resgate de plantas e plantio de vegetação nativa na fazenda Intermontes, município de Ribeirão Grande, SP. 2005. Tese (Doutorado em Recursos Florestais) - Escola Superior de Agricultura Luiz de Queiroz, Universidade de São Paulo, Piracicaba, 2005. Disponível em: <http://www.teses.usp.br/teses/ disponiveis/11/11150/tde-02062005-153506/>. Acessado em: 2011-10-26.

PICKETT, S. T. A.; COLLINS, S. L.; ARNESTO, J. J. A hierarchical consideration of causes and mechanisms of succession. Vegetatio, v. 69, n. 1-3, p. 109-114, 1987.

RODRIGUES, R. R.; BRANCALION, P. H. S.; ISERnHAGEN, I. (Eds.) Pacto para a restauração ecológica da Mata Atlântica: referencial dos conceitos e ações de restauração florestal. São Paulo: Instituto BioAtlântica, 2009a.p.115-118.

RODRIGUES, R.R.; GANDOLFI, S. Conceitos, tendências e ações para recuperação de florestas ciliares. In: RODRIGUES, R.R.; LEITÃO FILHO, H.F. (Eds.). Matas ciliares: conservação e recuperação. 2 ed. São Paulo: EDUSP/FAPESP, 2001. p. 235-248.

RODRIGUES, R. R.; GANDOLFI, S. Recuperação de formações ciliares: conceitos, tendências, modelos de implantação e recomendações práticas. In: RODRIGUES, R. R.; LEITÃO FILHO, H. F.(Ed.). Matas Ciliares: conservação e recuperação. 2 ed. São Paulo: EDUSP/FAPESP, 2001, p. 233-247

RODRIGUES, R.R.; GANDOLFI, S. Recomposição das florestas nativas: princípios gerais e subsídios para uma definição metodológica. Revista Brasileira de Horticultura Ornamental, Campinas, v.2 n.1, p. 4-15, 1996. 
RODRIGUES, R.R.; GANDOLFI, S. Restauração de florestas tropicais: subsídios para uma definição metodológica e indicadores de avaliação e monitoramento. In: Dias, L.E e Mello, J.W.V. (Eds) Recuperação de Áreas Degradadas.

Editora Folha de Viçosa Ltda. Viçosa, p.251, 1998.

STURION, J. A.; ANTUNES, B. M. A. Produção de mudas de espécies florestais. In: GALVÃO, A. P. M. Reflorestamento de propriedades rurais para fins produtivos e ambientais. Brasília: Embrapa, 2000. PAGINAS.

SWAINE, M. D.; WHITMORE, T.C. On the definition of ecological species groups in tropical rain forests. Vegetatio, v.75, n.1-2, p.81-86, 1988.
UGALDE, L. A. Comportamiento inicial de Acacia auriculiformis, Albizia falcataria, Calliandra calothyrsus, Leucaena leucocephala y Sesbania grandiflora em dos sítios em Costa Rica. In:BARROS, N. F. de. Florestas plantada no neotropico como fonte de energia. Universidade Federal de Viçosa, 1985, p.204-222.

VICENTINI, E. et al. Aspectos demográficos de espécies de Cecropia em fragmento de mata ciliar da usina hidrelétrica Rio Bonito, Santa Maria de Jetibá ES. Espírito Santo, 2008. Disponível em: http:// www.naturezaonline.com.br/natureza/conteudo/ pdf/04_VicentiniEetal_6672.pdf. Acessado em 20 out. de 2011 . 
